\title{
Risk Factors for Cervical Sagittal Malalignment after Cervical Laminoplasty
}

\author{
Sun Yoon, Jeong Yoon Park, Sung Uk Kuh, Dong-Kyu Chin, Keun Su Kim, Yong Eun Cho, \\ Kyung Hyun Kim
}

Department of Neurosurgery, Gangnam Severance Hospital, Spine and Spinal Cord Institute, Yonsei University College of Medicine, Seoul, Republic of Korea

Corresponding author: Kyung Hyun Kim

Department of Neurosurgery, Gangnam Severance Hospital, Spine and Spinal Cord Institute, Yonsei University College of Medicine, 211 Eonju-ro,

Gangnam-gu, Seoul 06273, Republic of Korea

Tel: +82-2-2019-3404

Fax: +82-2-3461-9229

E-mail: nskhk@yuhs.ac

Received: July 9, 2017

Revised: August 8, 2017

Accepted: August 16, 2017
Objective: Objective: To investigate the risk factors for cervical sagittal malalignment after cervical laminoplasty. Methods: The study included 41 patients who were treated with cervical laminoplasty from February 2006 to January 2010. The average follow-up period was 30.5 (range, 23-59 months). We divided the enrolled patients into three groups (Group A: C2 and C7 ligament preservation, Group B: C2 or C7 ligament preservation, Group C: Both C2 and C7 ligament excised). The patients' preoperative and postoperative clinical results were retrospectively reviewed using the Japanese Orthopedic Association (JOA) score for functional outcome. Radiography was performed before surgery and at the final follow-up, and the C2-7 Cobb angle, sagittal vertical axis (SVA) C2-7, range of motion (ROM), C2 tilt, C1-2 lordosis, and T1 tilt were measured to evaluate cervical alignment. Results: Change in SVA C2-7, C2-7 lordosis, C1-2 lordosis, C2 tilt, and ROM showed statistically significant differences in groups $B$ and $C$, but no statistically significant difference in group A for all these radiographic parameters. There was no statistically significant difference between preoperative and postoperative clinical outcome measurements for each group, as assessed by JOA scores ( $p=0.999,0.095$, and 0.111, respectively). In ANOVA, change in SVA C2-7 showed a statistically significant difference $(p=0.037)$ between the groups. The post-hoc analysis revealed a statistically significant difference between groups $A$ and $B(p=0.044)$ and between groups $A$ and $C(p=$ 0.011). Conclusion: We investigated SVA C2-7 as a factor of cervical sagittal alignment, and found that as the involvement of C2 and C7 ligamentous structures increased, SVA C2-7 also increased.

Key Words: Bone malalignment; Cervical vertebrae; Laminoplasty; Lordosis

\section{INTRODUCTION}

Over the decades, several effective surgical options have been developed such as anterior cervical discectomy or corpectomy and fusion, posterior cervical laminoplasty, and posterior cervical laminectomy and instrumented fusion for treating cervical spondylotic myelopathy (CSM), herniated cervical disc (HCD), and ossification of the posterior longitudinal ligament (OPLL). Many studies have been conducted to compare the outcomes of these techniques clinically and radiologically.

Recently, cervical sagittal balance has been identified as an important determinant of radiological outcomes following cervical surgery ${ }^{3,12)}$. However, few studies have investigated preand postoperative radiologic changes in cervical surgery and the risk factors for these changes, considering the relatively recent interest in the concept of sagittal alignment. Tang et al. $^{17)}$ played a pivotal role in highlighting the concept of cervical regional sagittal imbalance. They analyzed the relationships between radiographic parameters and health-related quality of life (HRQOL) score. Of several radiographic parameters, sagittal vertical axis (SVA) C2-7 showed a negative association with HRQOL scores after posterior cervical fusion ${ }^{17)}$. A previous study reported that posterior ligamentous structures attached to C2 and C7 played important roles in clinical outcome, but this study only evaluated clinical outcomes such as neck pain and did not examine the effects on the change in radiographic sagittal parameters between the preoperative and postoperative status $^{11)}$.

Thus, we hypothesized that the destruction of these uppermost and lowermost cervical stabilizing structures by various means, such as surgical techniques, and the level of pathologic lesion might have significant influences on cervical spinal sagittal alignment and balance. This study aimed to investigate the risk factors for postoperative cervical sagittal malalignment and sagittal imbalance. In particular, we focused on the preservation of $\mathrm{C} 2$ and $\mathrm{C} 7$ posterior ligamentous structures during surgery. 


\section{MATERIALS AND METHODS}

\section{Patients and Study Design}

Forty-one patients with HCD, CSM, and OPLL were included in this retrospective study. Each patient underwent a cervical laminoplasty at our hospital between February 2006 and January 2010 and provided informed consent. Patients with a history of previous spine surgery, congenital block vertebrae, acquired deformity due to infection, spinal cord tumor, and trauma were excluded.

Enrolled patients were divided into three groups depending on whether $\mathrm{C} 2$ and/or C7 ligamentous structures were preserved. Group A was composed of patients with both $\mathrm{C} 2$ and C7 ligamentous structures preserved. Group B comprised patients with either $\mathrm{C} 2$ or $\mathrm{C} 7$ ligamentous structure preserved. Group C consisted of patients without C2 or C7 ligamentous structures preserved.

\section{Measurement of Radiologic Parameters}

Preoperative and postoperative lateral standing radiographs were obtained using the protocol for the standard lateral cervical radiograph with the patients standing in a neutral position and instructed to look straight ahead with the knees locked ${ }^{17)}$. We measured several radiographic parameters and finally included six parameters ((1) SVA C2-7, (2) C2-7 Cobb angle, (3) C1-2 lordosis, (4) C2 tilt, (5) T1 tilt, and (6) range of motion [ROM]) in the statistical analyses. Detailed methods of measurement are as follows (Fig. 1):

(1) SVA C2-7 was defined as the distance of the C2 plumb line (extending from the centroid of the $\mathrm{C} 2$ vertebra) from the posterior superior end plate of $\mathrm{C} 7$.

(2) C2-7 lordosis was measured using usual sagittal Cobb angles. A positive value indicated lordotic alignment and a negative value indicated kyphotic alignment.

(3) C1-2 lordosis was calculated as a line connecting the anterior tubercle to the posterior margin of the $\mathrm{C} 1$ spinous process and the inferior endplate of $\mathrm{C} 2$.

(4) C2 tilt was the angle subtended by a line parallel to the posterior border of the odontoid and a vertical line. A negative value was defined when the superior tip of the line along the posterior odontoid was posterior to the vertical line.

(5) T1 tilt was measured as the angle between a horizontal line and the superior endplate of $\mathrm{T} 1$ in a standing lateral radiograph.

(6) ROM of the cervical spine was assessed as the difference between a lordotic or kyphotic C2-7 Cobb angle measured during extension and the same angle during flexion.

\section{Clinical Outcome Measurement}

Functional outcomes were evaluated using the Japanese Orthopedic Association (JOA) score. This score was measured at the preoperative stage and at the last follow-up visit to the outpatient clinic and the difference between at the two scores was considered the clinical outcome of the surgery.

\section{Statistical Analysis}

Data analysis was performed using SPSS version 20.0 (IBM Corp., Armonk, NY, USA). Alpha was set at 0.05 for each analysis. Repeated-measure analysis of variance (ANOVA) with posthoc (Bonferroni) corrections was used to determine the difference in clinical and radiographic parameters between the three groups. The paired t-test was used to determine whether there was a statistically significant difference in the mean and standard deviation of the preoperative, postoperative, and change values of each parameter.

\section{RESULTS}

\section{Patients' Demographics and Overall Results}

Forty-one patients (33 male, 8 female) were enrolled in the

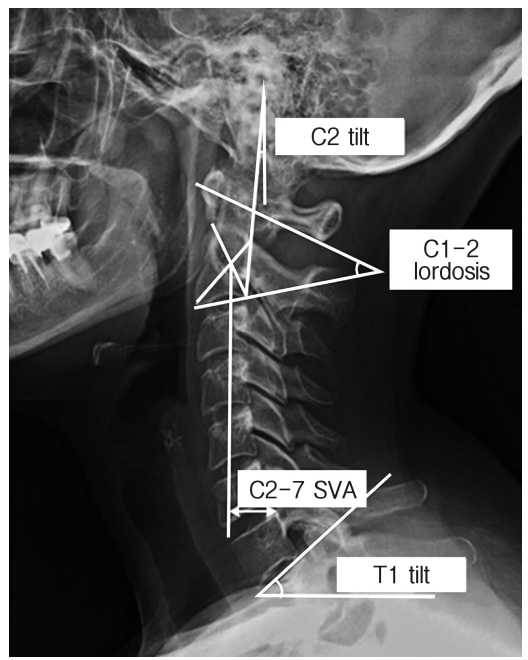

Fig. 1. The distance of the $\mathrm{C} 2$ plumb line from the posterior superior end plate of C7 (Sagittal vertical axis [SVA] C2-7). A line connecting the anterior tubercle to the posterior margin of the $\mathrm{Cl}$ spinous process and the inferior endplate of $\mathrm{C} 2$ ( $\mathrm{Cl}-2$ lordosis). The angle subtended by a line parallel to the posterior border of the odontoid and a vertical line (C2 tilt). Measured as the angle between a horizontal line and the superior endplate of $\mathrm{Tl}$ in a standing lateral radiograph (T1 tilt). 
Table 1. Comparative data among the groups with regard to demographics and perioperative clinical and radiological outcomes

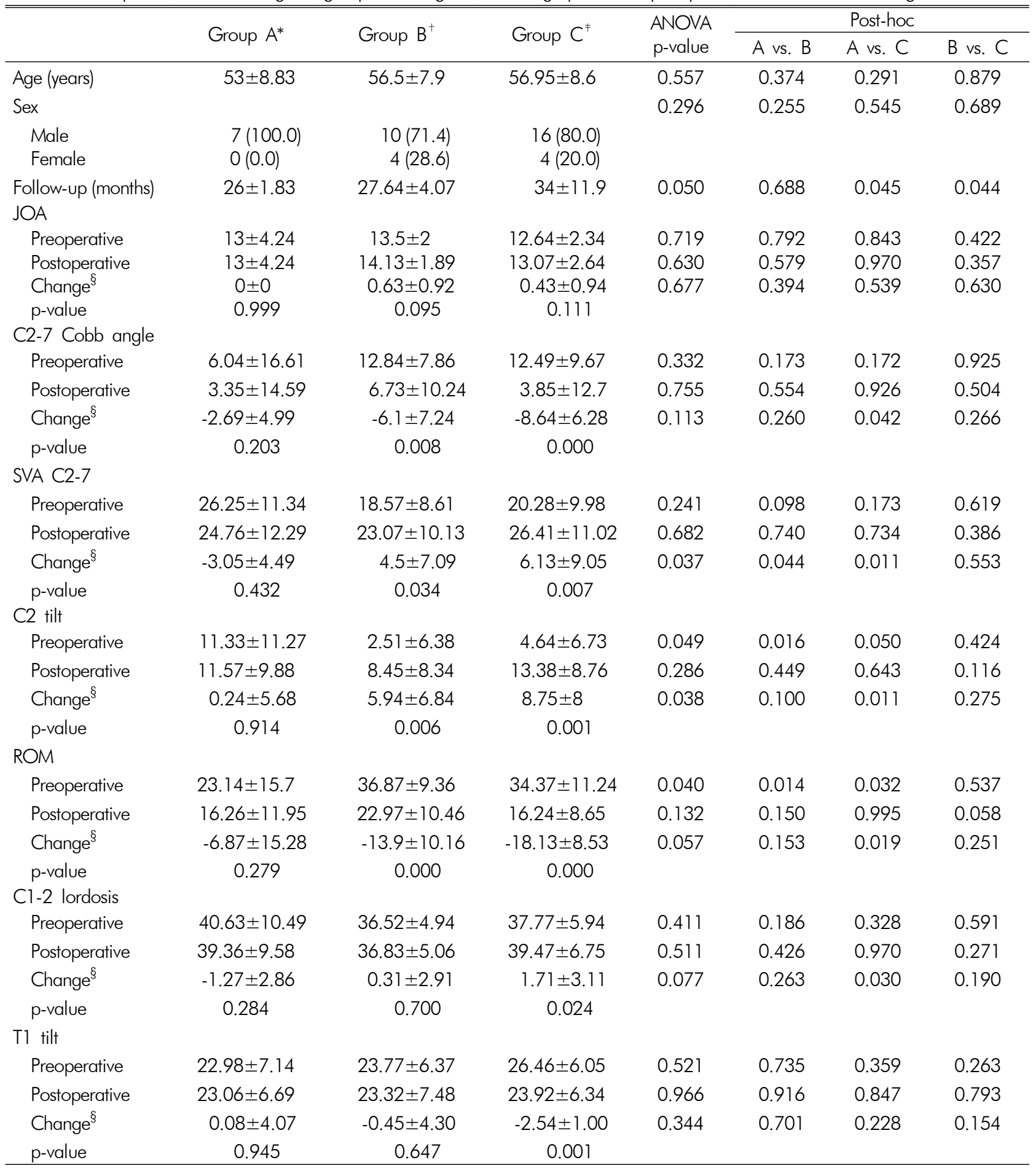

The data is presented as mean \pm standard deviation or number $(\%)$.

In accordance with the sex ratio, the chi-square test was used for statistical analysis. Confirmation age, sex, duration and change between pre- and postoperative values (Independent two-sample t-test and chi-square test).

p-value: two-sample paired t-test. ANOVA with post-hoc analysis.

${ }^{*}$ Group A: C2 and C7 ligament preservation. ${ }^{+}$Group B: C2 or C7 ligament preservation. ${ }^{*}$ Group C: Both C2 and C7 ligament excised. ${ }^{\S}$ Change in variables: Postoperative value - Preoperative value.

ANOVA: analysis of variance; JOA: Japanese Orthopedic Association; SVA: sagittal vertical axis; ROM: range of motion. 
study. The mean patient age was 56.1 (range, 39-77 years) and the mean follow-up period was 30.5 (range, 23-59 months). Patients were divided into three groups (group A, B, and C) comprising 7, 14, and 20 patients, respectively. There was no statistically significant difference in age and sex between the groups. Patients' demographic data are summarized in Table 1.

Change in SVA C2-7, C2-7 lordosis, C1-2 lordosis, C2 tilt, and $\mathrm{ROM}$ showed statistically significant differences in group B and C, except for C1-2 lordosis that showed no statistically significant difference in group $B(p=0.700)$. However, there was no significant difference in group A for all these radiographic parameters (Table 1).

\section{Comparative Analysis Between Groups}

There was no statistically significant difference between preoperative and postoperative clinical outcome measurements using JOA scores (Group A, p=0.999; Group B, p=0.095, Group $\mathrm{C}, \mathrm{p}=0.111$ ) (Table 1). Moreover, no significant differences were observed between the groups in the ANOVA $(p=0.677)$ and post hoc analysis (Group A vs. B, p=0.394, Group A vs. C, p= 0.539, Group B vs. C, $\mathrm{p}=0.630$ ).

In ANOVA, change in SVA C2-7 showed a significant difference $(\mathrm{p}=0.037)$ between the groups. The post-hoc analysis revealed significant differences between groups $A$ and $B(p=0.044)$ and between groups $\mathrm{A}$ and $\mathrm{C}(\mathrm{p}=0.011)$.

A significant difference was not found in change in $\mathrm{C} 2-7$ lordosis between the groups in ANOVA ( $\mathrm{p}=0.113)$. Based on the post hoc analysis, a significant difference was only noted between Groups A and C ( $\mathrm{p}=0.042)$.

In ANOVA, preoperative, postoperative, and change in C1-2 lordosis values showed no significant differences between the groups, although the post-hoc analysis showed a significant difference between group A and $\mathrm{C}(\mathrm{p}=0.030)$.

In ANOVA, C2 tilt showed statistically significant differences for preoperative and change in $\mathrm{C} 2$ tilt values $(\mathrm{p}=0.049$ and $\mathrm{p}=$ 0.038 , respectively); however, no significant difference was noted for postoperative C2 tilt $(\mathrm{p}=0.286)$. The post-hoc analysis showed a significant difference for change in C2 tilt between groups $\mathrm{A}$ and $\mathrm{C}(\mathrm{p}=0.011)$.

Change in T1 tilt was not significantly different between groups $\mathrm{A}$ and $\mathrm{B}(\mathrm{p}=0.945$ and $\mathrm{p}=0.647$, respectively), but showed a significant difference in group $\mathrm{C}(\mathrm{p}=0,001)$. However, change in T1 tilt showed no significant differences in the ANOVA or post hoc analysis.

Change in ROM was significantly different between groups $\mathrm{B}$ and $\mathrm{C}$. In ANOVA, preoperative ROM showed a statistically significant difference $(\mathrm{p}=0.040)$ among the three groups. However, change in ROM showed no significant difference $(\mathrm{p}=$ 0.057). In the post-hoc analysis, change in ROM was significantly different between Group A and C. However, ANOVA showed no significant difference in the change in ROM between the groups.

\section{Correlative Analysis and Multiple Linear Regre- ssion Analysis for Postoperative Cenvical Sagittal Malalignment}

As a representative parameter of cervical sagittal imbalance, change in SVA C2-7 was evaluated with correlative analysis. There were no statistically significant differences in patient age,

Table 2. Correlation analysis of various clinical and radiological variables with sagittal imbalance (Delta SVA C2-7)

\begin{tabular}{|c|c|c|}
\hline Variable & $\mathrm{R}$ or mean $\pm \mathrm{SD}$ & p-value \\
\hline Age & -0.008 & 0.963 \\
\hline Sex & & 0.772 \\
\hline Female & $4.2 \pm 8.37$ & \\
\hline Male & $3.19 \pm 8.64$ & \\
\hline Follow-up & -0.068 & 0.672 \\
\hline Group & & 0.037 \\
\hline Group A & $-3.05 \pm 4.48$ & \\
\hline Group B & $4.49 \pm 7.08$ & \\
\hline Group C & $6.13 \pm 9.04$ & \\
\hline \multicolumn{3}{|l|}{$\mathrm{JOA}$} \\
\hline Preoperative & -0.182 & 0.395 \\
\hline Postoperative & -0.210 & 0.325 \\
\hline Change & -0.114 & 0.596 \\
\hline \multicolumn{3}{|c|}{ C2-7 Cobb angle } \\
\hline Preoperative & 0.088 & 0.584 \\
\hline Postoperative & -0.249 & 0.117 \\
\hline Change & -0.59 & \\
\hline \multicolumn{3}{|l|}{ SVA C2 } \\
\hline Postoperative & 0.463 & 0.002 \\
\hline \multicolumn{3}{|l|}{ C2 tilt } \\
\hline Preoperative & -0.281 & 0.075 \\
\hline Postoperative & 0.49 & 0.001 \\
\hline Change & 0.849 & \\
\hline \multicolumn{3}{|l|}{$\mathrm{Cl}$ inclination } \\
\hline Preoperative & 0.270 & 0.088 \\
\hline Postoperative & -0.182 & 0.254 \\
\hline Change & -0.709 & \\
\hline \multicolumn{3}{|l|}{ T1 tilt } \\
\hline Preoperative & -0.025 & 0.876 \\
\hline Postoperative & 0.164 & 0.307 \\
\hline Change & 0.331 & 0.034 \\
\hline
\end{tabular}

There was a statistically significant difference between the groups and a correlation between change of SVA C2-7 and change in C2-7 Cobb angle, postoperative and change of C2 tilt values, and change in $\mathrm{T} 1$ tilt. We designed a multivariate linear regression analysis for further verification.

JOA: Japanese Orthopedic Association; SVA: sagittal vertical axis. *Statistically significant values. 
sex, and JOA scores between the groups.

Change in C2-7 lordosis and C2 tilt showed a significant moderate negative correlation $(\mathrm{R}=-0.59, \mathrm{R}=0.849, \mathrm{p}<0.001)$. Change in T1 tilt showed a significant moderate correlation with change in SVA C2-7 $(\mathrm{R}=0.331, \mathrm{p}=0.034)$ (Table 2).

\section{DISCUSSION}

Currently, the importance of cervical sagittal alignment has been acknowledged and sagittal imbalance after posterior surgery on the cervical spine has been considered a major source of pain and disability ${ }^{33}$. Sakai et al. ${ }^{13)}$ suggested that preoperative cervical sagittal imbalance is a powerful predictor of kyphotic deformity after cervical laminoplasty and is related to poor postoperative clinical outcomes.

Suk et al. ${ }^{10)}$ reported a significant decrease in cervical lordosis after laminoplasty and that the incidence of kyphotic change was significantly higher in the decreased preoperative cervical lordosis group (Cobb angle $\left.<10^{\circ}\right)$. This study also showed the loss of cervical lordosis by measuring the change in C2-7 Cobb angles and comparing preoperative and postoperative values (Group A: $-2.69 \pm 4.99$, B: $-6.1 \pm 7.24$, C: $-8.64 \pm 6.28$ ). Although there were no statistically significant differences in change in C2-7 Cobb angle between the three groups with the ANOVA, change in C2-7 Cobb angle revealed a strong negative correlation with change in SVA C2-7. Thus, we concluded that the postoperative loss of cervical lordosis is an important risk factor for postoperative cervical sagittal imbalance.

Some previous studies have shown that C2-7 paraspinal muscle and ligament preservation can reduce the postoperative loss of cervical lordosis. Sakaura et al. ${ }^{14)}$ reported the 8-years' follow-up data of 20 patients after cervical laminoplasty from the C3 to C6 level and the patients did not show any postoperative kyphotic change. Michael et al. ${ }^{8)}$ reported a more significant postoperative loss of cervical lordosis when C3 laminas were included as compared to when $\mathrm{C} 4$ laminas were included and C3 laminas excluded. C6/7 lesional cervical alignment also plays important roles in the maintenance of cervical sagittal alignment ${ }^{1}$. Sakaura et $\mathrm{al}^{15}{ }^{15}$ reported that the funicular structures of the nuchal ligament attached to $\mathrm{C} 6 / 7$ played important roles in decreasing the segmental alignment of $\mathrm{C} 6 / 7$ postoperatively. Lin et $\mathrm{al}^{7}$. introduced a laminoplasty technique with unilateral muscle-ligament complex preservation and compared this procedure with the traditional open-door laminoplasty. Their modality showed superiority in maintaining cervical sagittal alignment, including SVA C2-7 and C3-7 cobb angle measurement. These previous studies indicate that the paraspinal muscles and nuchal ligament play important roles in the maintenance of cervical lordosis. Furthermore, C2 and C7 are the core attachment points of the paraspinal muscles and nuchal ligament for maintaining cervical lordosis. Our study results indicate a relationship between $\mathrm{C} 2 / 7$ musculoligamentous structure preservation and cervical alignment. Consequently, we assumed that $\mathrm{C} 2-\mathrm{C} 7$ ligament disruption during surgery can influence the postoperative loss of cervical lordosis, sagittal imbalance, and poor clinical outcomes ${ }^{2,9,14)}$.

We considered the change in SVA C2-7 as a representative parameter of cervical sagittal imbalance. Previous studies focused on the C2-7 loss of lordosis as a radiographic parameter for evaluating postoperative change. A few studies have evaluated the relationship of SVA C2-7 with cervical sagittal imbalance, although many studies have suggested a negative relationship between SVA C2-7 and clinical outcomes ${ }^{18,20)}$.

Comparing the change in SVA C2-7, C2-7 lordosis, and T1 tilt with differences among groups and considering C2-7 ligament structure disruption that causes cervical spine destabilization and loss of cervical lordosis, it is difficult to elucidate which of these first results in the loss of cervical lordosis. However, it can be ascertained that changes in C2 tilt, T1 tilt, and SVA $\mathrm{C} 2$ are correlated with and ultimately affect the forward pitch of cervical sagittal balance.

T1 slope has been used as an important parameter for predicting surgical outcomes after posterior cervical surgery, and some studies have reported a correlation between T1 slope and C2-7 lordosis and SVA C2- $7^{4-6,10,19)}$. Patients with a higher preoperative T1 slope were more likely to exhibit a postoperative loss of $\mathrm{C} 2-7$ lordosis, which was greater at the degree when it occurred. However, postoperative kyphosis did not occur more frequently in patients with higher T1 slopes ${ }^{3}$. Our study revealed that T1 tilt has a negative correlation with change in SVA C2-7. It could be suggested that a high T1 tilt results in a large C2-7 lordosis and that posterior cervical spine surgery and disruptions of $\mathrm{C} 2,7$ musculoskeletal structures lead to the forward shifting of the center of gravity and change of SVA C2-7. Thus, these three parameters: SVA C2-7, T1 tilt, and SVA C2-7 have a complex organic correlation.

The limitations of this study include the small number of enrolled patients and their further division into three separate groups. Moreover, the follow-up periods of each group showed some differences. Therefore, a larger number of patients and longterm follow-up seem necessary for more definitive conclusions.

We suggest that the increase in C2 and T1 tilt could result from C2-7 supra and interspinous ligament destabilization, which might influence the postoperative loss of cervical lordosis and increased C2 SVA. Additional multivariate linear regression analysis revealed that the loss of cervical lordosis and increase of $\mathrm{C} 2$ and $\mathrm{T} 1$ tilt are significant factors for increased change of SVA C2-7 after cervical laminoplasty.

\section{CONCLUSION}

In analyzing $\mathrm{C} 2$ and/or $\mathrm{C} 7$ ligament structure involvements during the surgery, we found that $\mathrm{C} 2$ and $\mathrm{C} 7$ preservation during laminoplasty plays an important role in minimizing the preoperative-postoperative change of SVA C2-7, which means maintenance of cervical sagittal alignment. 


\section{CONFLICTS OF INTEREST}

No potential conflict of interest relevant to this article was reported.

\section{ACKNOWLDGEMENTS}

This research was supported by a grant of the Korean Health Technologiy R\&D Project through the Korea Health Industry Development Institute (KHIDI), Funded by the Ministry of Health \& Welfare, Republic of Korea(grant number:HC15C1320).

\section{REFERENCES}

1. Hosono N, Sakaura H, Mukai Y, Ishii T, Yoshikawa H: En bloc laminoplasty without dissection of paraspinal muscles. J Neurosurg Spine 3:29-33, 2005

2. Iizuka $H$, Nakajima T, lizuka Y, Sorimachi Y, Ara T, Nishinome $\mathrm{M}$, et al.: Cervical malalignment after laminoplasty: relationship to deep extensor musculature of the cervical spine and neurological outcome. J Neurosurg Spine 7:610-614, 2007

3. Kim B, Yoon DH, Ha Y, Yi S, Shin DA, Lee CK, et al.: Relationship between T1 slope and loss of lordosis after laminoplasty in patients with cervical ossification of the posterior longitudinal ligament. Spine J 16:219-225, 2016

4. Kim TH, Lee SY, Kim YC, Park MS, Kim SW: T1 slope as a predictor of kyphotic alignment change after laminoplasty in patients with cervical myelopathy. Spine (Phila Pa 1976) 38: E992-E997, 2013

5. Knott PT, Mardjetko SM, Techy F: The use of the T1 sagittal angle in predicting overall sagittal balance of the spine. Spine J 10:994-998, 2010

6. Lee SH, Son ES, Seo EM, Suk KS, Kim KT: Factors determining cervical spine sagittal balance in asymptomatic adults: correlation with spinopelvic balance and thoracic inlet alignment. Spine J 15:705-712, 2015

7. Lin S, Zhou F, Sun Y, Chen Z, Zhang F, Pan S: The severity of operative invasion to the posterior muscular-ligament complex influences cervical sagittal balance after open-door laminoplasty. Eur Spine J 24:127-135, 2015

8. Michael KW, Neustein TM, Rhee JM: Where should a laminoplasty start? The effect of the proximal level on post-laminoplasty loss of lordosis. Spine J 16:737-741, 2016

9. Nolan JP, Jr., Sherk HH: Biomechanical evaluation of the exten- sor musculature of the cervical spine. Spine (Phila Pa 1976) 13: 9-11, 1988

10. Park JH, Cho CB, Song JH, Kim SW, Ha Y, Oh JK: T1 slope and cervical sagittal alignment on cervical CT radiographs of asymptomatic persons. J Korean Neurosurg Soc 53:356-359, 2013

11. Riew KD, Raich AL, Dettori JR, Heller JG: Neck pain following cervical laminoplasty: does preservation of the C2 muscle attachments and/or C7 matter? Evid Based Spine Care J 4:42-53, 2013

12. Sakai K, Yoshii T, Hirai T, Arai Y, Shinomiya K, Okawa A: Impact of the surgical treatment for degenerative cervical myelopathy on the preoperative cervical sagittal balance: a review of prospective comparative cohort between anterior decompression with fusion and laminoplasty. Eur Spine J 26:104-112, 2017

13. Sakai K, Yoshii T, Hirai T, Arai Y, Torigoe I, Tomori M, et al.: Cervical sagittal imbalance is a predictor of kyphotic deformity after laminoplasty in cervical spondylotic myelopathy patients without preoperative kyphotic alignment. Spine (Phila Pa 1976) 41:299-305, 2016

14. Sakaura H, Hosono N, Mukai Y, Iwasaki M, Yoshikawa H: C3-6 laminoplasty for cervical spondylotic myelopathy maintains satisfactory long-term surgical outcomes. Global Spine J 4:169-174, 2014

15. Sakaura H, Hosono N, Mukai Y, Oshima K, Iwasaki M, Yoshikawa $\mathrm{H}$ : Preservation of the nuchal ligament plays an important role in preventing unfavorable radiologic changes after laminoplasty. J Spinal Disord Tech 21:338-343, 2008

16. Suk KS, Kim KT, Lee JH, Lee SH, Lim YJ, Kim JS: Sagittal alignment of the cervical spine after the laminoplasty. Spine (Phila Pa 1976) 32:E656-E660, 2007

17. Tang JA, Scheer JK, Smith JS, Deviren V, Bess S, Hart RA, et al.: The impact of standing regional cervical sagittal alignment on outcomes in posterior cervical fusion surgery. Neurosurgery 71:662-669; discussion 669, 2012

18. Tauchi R, Lee SH, Kim JY, Kim YC, Peters C, Imagama S, et al.: Postoperative severe headache following cervical posterior surgical fixation from C2 distally. Asian Spine J 10:728-733, 2016

19. Weng C, Wang J, Tuchman A, Wang J, Fu C, Hsieh PC, et al.: Influence of T1 slope on the cervical sagittal balance in degenerative cervical spine: an analysis using kinematic MRI. Spine (Phila Pa 1976) 41:185-190, 2016

20. Yeh KT, Lee RP, Chen IH, Yu TC, Peng CH, Liu KL, et al.: The midterm surgical outcome of modified expansive open-door laminoplasty. Biomed Res Int 2016:8069354, 2016 